\title{
Case report of infantile cholestasis with liver hemangioma
}

\begin{abstract}
Neonatal cholestasis is far less common than unconjugated hyperbilirubinemia. Despite its relative infrequency, neonatal cholestasis is always pathologic. Therefore, the clinician should consider the possibility of cholestasis in a jaundiced infant. This case was a 50days girl with cholestasis and abdominal distention due to huge hemangioma.
\end{abstract}

Keywords: cholestasis, hyperbilirubinemia, infants, hepatic neoplasms, liver enzymes
Volume 3 Issue 6 - 2017

\author{
Pantea Tajik \\ Department of Pediatric Gastroenterohepatology, \\ Amiralmomenin Hospital, Iran
}

\begin{abstract}
Correspondence: Pantea Tajik, MD, Assistant Prof. of Pediatric Gastroenterohepatology in Amiralmomenin hospital , Semnan, Iran,Email patal5000@gmail.com
\end{abstract}

Received: August 06, 2017| Published: November 27, 2017

\section{Introduction}

Cholestasis refers to impedance to bile acid formation or flow and is evidenced by elevated conjugated or direct hyperbilirubinemia. An elevated direct bilirubin as greater than 1.0 if the total bilirubin is less than 5.0; and greater than $20 \%$ of the total bilirubin if the total bilirubin is greater than 5.0. Unlike neonatal unconjugated (indirect) hyperbilirubinemia, which is more frequently physiologic rather than pathologic, conjugated hyperbilirubinemia should always be viewed as abnormal and should not be ignored. Early recognition of cholestasis in infants and prompt diagnosis of the underlying disorder are imperative to identify those disorders that respond to specific treatment. It is therefore recommended that any jaundiced infant have total and direct bilirubin checked at 2 to 3 weeks of age. ${ }^{1-7}$

\section{More common causes of neonatal cholestasis ${ }^{6}$}

Obstructive: Biliary Atresia, Choledochal Cyst, Hemangioma, Hepatoblastoma, Gallstones/Biliary Sludge, Inspissated Bile (Due To Hemolysis), Alagille Syndrome (Biliary Hypoplasia), Cystic Fibrosis.

Hepatocellular: Idiopathic neonatal hepatitis, Viral (CMV, HIV), Bacterial, Urinary tract infection, Sepsis, Syphilis.

Genetic/Metabolic: 1-Antitrypsin deficiency, Cystic fibrosis, Hypothyroidism, hypopituitarism, Tyrosinemia, Galactosemia, Progressive familial intrahepatic cholestasis, Cystic fibrosis.

Toxic: Total parenteral nutrition.

Drugs: Most infants with neonatal cholestasis present with jaundice, dark urine, a colic stools, and varying degrees of hepatomegaly. It is necessary to evaluate infants for evidence of congenital anomalies, splenomegaly, skin rash, and neurologic signs. Cholestatic jaundice because of hemangioma -related bile duct compression. ${ }^{7-8}$

Hepatic hemangioma, also known as hepatic venous malformations, is benign non-neoplastic hyper vascular liver lesions. They are frequently diagnosed as an incidental finding on imaging, and most patients are asymptomatic. From a radiologic perspective, it is important to differentiate hemangioma from hepatic neoplasms. Hepatic hemangioma is thought to be congenital in origin, nonneoplastic, and is almost always of the cavernous subtype. Blood supply is predominantly hepatic arterial, similar to other liver tumors. A peripheral location within the liver is most common. ${ }^{8-10}$

\section{Case report}

A 50days' girl with birth weight $3 \mathrm{~kg}$ now was $5 \mathrm{~kg}$, NVD delivery without any complication. The infant was icteric in 35days. In 40days she admitted in ward so in history every things was normal and her mother was normal and she never use any drugs or smoking. In physical examination the infant was so generalized icteric abdominal distention, umbilical hernia, edema and respiratory distress. Liver was huge and span $10 \mathrm{~cm}$, spleen was palpable.

\section{The laboratory tests were}

Blood investigations revealed anemia $(\mathrm{Hb} 7 \mathrm{gm} / \mathrm{dl})$, normal total and differential leukocyte count and severe thrombocytopenia (platelet count 70,000/ $\mu \mathrm{L}$ ), AST 200, ALT 450, ALK 678, GGT 150, T. Billi $10 \mathrm{mg} / \mathrm{dl}$, D $6 \mathrm{mg} / \mathrm{dl}$, PT $16 \mathrm{sec}$, PTT $30 \mathrm{sec}$, INR $2 \mathrm{U} / \mathrm{A}-\mathrm{U} / \mathrm{C}$ were normal. Other biochemistry lab test was normal. TORCH study, viral marker hepatitis, TFT, B/C and metabolic study were normal. HIDA scan was normal. Chest $\mathrm{X}$ ray was normal. Brain and pelvic CT were normal. In sonography typically well-defined hyperechoic lesions was seen in left lobe. So contrast abdominal spiral CT was done and in CT well defined enhance lesion was seen due to liver hemangioma (Figure 1). So surgical consult was done then the infant transfer to surgery ward and the left lobe of liver was lobectomy and after 2weeks the T. Billi 3D1 and after 2months the infant was good and lab test were normal.

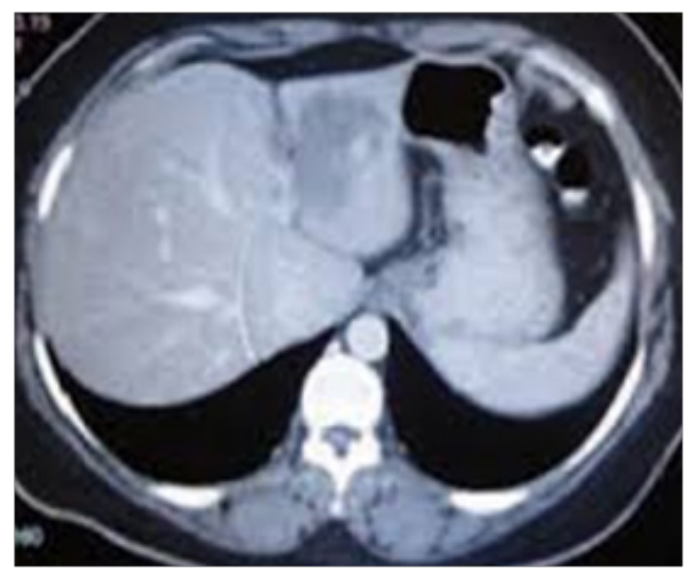

Figure I CT well defined enhance lesion was seen due to liver hemangioma. 


\section{Discussion}

Cholestasis refers to impedance to bile acid formation or flow and is evidenced by elevated conjugated or direct hyperbilirubinemia. An elevated direct bilirubin as greater than 1.0 if the total bilirubin is less than 5.0; and greater than $20 \%$ of the total bilirubin if the total bilirubin is greater than 5.0. Unlike neonatal unconjugated (indirect) hyperbilirubinemia, which is more frequently physiologic rather than pathologic, conjugated hyperbilirubinemia should always be viewed as abnormal and should not be ignored.

Urinary tract infections are an important cause of cholestasis in otherwise well-appearing infants, so urinalysis and culture should be routinely included in the initial stages of evaluation. The newborn screen results should be reviewed, with particular attention to the possibility of hypothyroidism, galactosemia, and cystic fibrosis. Urine-reducing substance (for galactosemia) and additional infant testing for thyroid function can also be considered. Knowledge of any maternal-infant blood group incompatibility is useful; as hemolysis can contribute to cholestasis via inspissate bile. CBC may provide evidence of sepsis or hemolysis. Liver enzymes ALT, AST, alkaline phosphatase, and GGT are included in the evaluation, although none is capable of diagnosing a particular condition. Protime/INR and albumin are tests of hepatic protein synthetic function. ${ }^{1-10}$

Hepatobiliary ultrasonography can make or exclude the diagnosis of choledochal cyst, as well as suggest (but not diagnose or rule out) biliary atresia. Hepatobiliary scintigraphy (e.g., HIDA scan), after 3 to 5days of premedication with Phenobarbital, is used by many to exclude biliary atresia, if the test is negative (isotope passing from the liver into the intestine). ${ }^{9}$

Hepatic hemangioma is thought to be congenital in origin, nonneoplastic, and is almost always of the cavernous subtype. Blood supply is predominantly hepatic arterial, similar to other liver tumors. A peripheral location within the liver is most common. Cholestatic jaundice because of hemangioma-related bile duct compression. ${ }^{11}$

\section{Sub types}

i. Typical hepatic hemangioma.

ii. Atypical hepatic hemangioma.

iii. Giant hepatic hemangioma.

iv. Flash filling hepatic hemangioma: can account for up to $16 \%$ of all hepatic hemangioma.

v. Slow fill hepatic hemangioma: can account for around $8-16 \%$ of all hemangioma. ${ }^{12}$

vi. Calcified hepatic hemangioma.

vii. Hyalinised/scleroses hepatic hemangioma.

viii. Other unusual imaging patterns.

ix. Hepatic hemangioma with capsular retraction.

x. Hepatic hemangioma with surrounding regional nodular hyperplasia.

xi. Hepatic hemangioma with fatty infiltration.

xii. Pedunculated hepatic hemangioma.

xiii. Cystic hepatic hemangioma: rare. xiv. Fluid-fluid level containing hepatic hemangioma: rare.

The presence of a few hepatic hemangiomas in the liver is not uncommon, but rarely a large number hepatic hemangioma may occur. ${ }^{12}$ Most hemangioma is relatively well defined. The dynamic enhancement pattern is related to the size of its vascular space.

Features of typical lesions include:

a. Noncontract: Often hypo attenuating relative to liver parenchyma.

b. Arterial phase: Typically show discontinuous, nodular, peripheral enhancement (small lesions may show uniform enhancement).

c. Portal venous phase: Progressive peripheral enhancement with more centripetal fill-in.

d. Delayed phase: Further irregular fill-in and therefore iso- or hyper-attenuating to liver parenchyma. ${ }^{11-12}$ They are benign lesions.

\section{Conclusion}

Recommendations for patients with no known risk factors for hepatic malignancy can range from center to center from performing confirmatory examinations (MR imaging, triphasic CT or scintigraphy) to considering follow-up ultrasound in 6months to confirm stability, to performing no further imaging evaluation. Some authors propose surgical resection for patients with progressive abdominal pain in combination with size greater than $5 \mathrm{~cm} .{ }^{13,14}$ So we should know one of the causes of cholestasis is liver hemangioma with pressure in bile duct that can see in sonography and abdominal $\mathrm{CT}$ and with surgery the symptom was removed.

\section{Acknowledgements}

None.

\section{Conflicts of interest}

Author declares that there is no conflict of interest.

\section{References}

1. Moyer V, Freese DK, Whitington PF, et al. Guideline for the evaluation of cholestatic jaundice in infants: Recommendations of the North American Society for Pediatric Gastroenterology, Hepatology and Nutrition. $J$ Pediatr Gastroenterol Nutr. 2004;39(2):115-128.

2. Mack CL, Sokol RJ. Unraveling the pathogenesis and etiology of biliary atresia. Pediatr Res. 2005;57(5 Pt 2):87R-94R.

3. Zhang DY, Sabla G, Shivakumar P, et al. Coordinate expression of regulatory genes differentiates embryonic and perinatal forms of biliary atresia. Hepatology. 2004;39(4):954-962.

4. Davenport MA. Challenge on the use of the words embryonic and perinatal in the context of biliary atresia. Hepatology. 2005;41(2):403-404.

5. Muise AM, Turner D, Wine E, et al. Biliary atresia with choledochal cyst: Implications for classification. Clin Gastroenterol Hepatol. 2006;4(11):1411-1414.

6. Balistreri WF, Bezerra JA, Ryckman FC. Biliary atresia and other disorders of the extrahepatic bile ducts. In: Suchy FJ, Sokol RJ, editors. Liver Disease in Children. Philadelphia, USA: Lippincott Williams \& Wilkins; 2001. p. 253-274. 
7. Aspelund G, Ling SC, Ng V, et al. A role for laparoscopic approach in treatment of biliary atresia and choledochal cysts. J Pediatr Surg. 2007;42(5):869-872.

8. Oto A, Kulkarni K, Nishikawa R, et al. Contrast enhancement of hepatic hemangiomas on multiphase MDCT: Can we diagnose hepatic hemangiomas by comparing enhancement with blood pool? AJR Am J Roentgenol. 2010;195(2):381-386.

9. Vilgrain V, Boulos L, Vullierme MP, et al. Imaging of atypical hemangiomas of the liver with pathologic correlation. Radiographics. 2000;20(2):379-397.

10. Jang HJ, Kim TK, Lim HK. Hepatic hemangioma: atypical appearances on CT, MR imaging, and sonography. AJR Am J Roentgenol. 2003;180(1):135-141.
11. Sica GT, Ji H, Ros PR. CT and MR imaging of hepatic metastases. AJR Am J Roentgenol. 2000;174(3):691-698.

12. Focal Liver Lesions: Detection, Characterization, Ablation. Springer.

13. Leifer DM, Middleton WD, Teefey SA, et al. Follow-up of patients at low risk for hepatic malignancy with a characteristic hemangioma at US. Radiology. 2000;214(1):167-172.

14. Erdogan D, Busch OR, Van Delden OM, et al. Management of liver hemangiomas according to size and symptoms. J Gastroenterol Hepatol. 2007;22(11):1953-1958. 